\title{
Methods for Testing Transport Models Second Year Annual Report
}

\author{
C. Singer and D. Cox \\ Departments of Nuclear Engineering and Statistics \\ University of Illinois at Urbana-Champaign
}

November 14, 1991

\begin{abstract}
Substantial progress has been made over the past year on six aspects of the work supported by this grant. As a result, we have in hand for the first time a fairly complete set of transport models and improved statistical methods for testing them against large databases. We also have initial results of such tests. These results indicate that careful application of presently available transport theories can reasonably well reproduce a remarkably wide variety of tokamak data. The particular results obtained so far are as follows. (1) A transport code emulator has been constructed by fitting SADLUR code input $\rightarrow$ output results with a linear regression plus an optimized random function. This result allows orders of magnitude reduction in the computational burden required to find confidence contours for a few a priori undetermined parameters in theoretical transport models. (2) The code emulator was used to find the probabilities that combined confinement data from more than one tokamak allow adjustable parameters (corresponding physically to turbulence saturation amplitudes) to lie within certain limits. To our knowledge, this represents the first statistically rigorous attempt to calibrate theorybased transport models against a sizeable tokamak data base. (3) Improved statistical methods were applied to deal with the expectation that results from a given tokamak would be more correlated than results from different machines. Results from the statistical model
\end{abstract}


adopted were found to be consistent with this expectation. (4) A model corresponding to a particular point in the set of possible turbulence saturation levels was used to simulate a wide variety of profile data. The very same model reproduced a variety of global confinement scaling results, including the machine-dependence of some of the confinement scaling exponents. (5) A sizeable body of information on the adequacy of our standard boundary conditions model has been accumulated and evaiuated. This model has been adequate for our investigations to date, but it will need improvement before long. (6) A new survey of available theoretical transport fluxes has been documented, using a formulation which defines a self-consistent method for integrating the approaches of various authors.

\section{DISCLAIMER}

\footnotetext{
This report was prepared as an account of work sponsored by an agency of the United States Government. Neither the United States Government nor any agency thereof, nor any of their employees, makes any warranty, express or implied, or assumes any legal liability or responsibility for the accuracy, completeness, or usefulness of any information, apparatus, product, or process disclosed, or represents that its use would not infringe privately owned rights. Reference herein to any specific commercial product, process, or service by trade name, trademark, manufacturer, or otherwise does not necessarily constitute or imply its endorsement, recommendation, or favoring by the United States Government or any agency thereof. The views and npinions of authors expressed herein do not necessarily state or reflect those of the United States Government or any agency thereof.
} 


\section{Introduction}

This report documents progress made on "Methods of Testing Transport Models," a grant jointly funded by Applied Plasma Physics and Confinement Systems. Since our last annual report, a number of projects which were at earlier stages of development have matured to the point of concrete application. An overview of these projects is given in the above abstract. Here, we describe each in more detail, and reference how the results have been documented.

\section{Results}

\subsection{Transport Code Emulator}

The basic idea of replacing an expensive computer code with a functional parameterization of its input/output relation has been known for some time in the controlled fusion area (e.g. [1]). The special features of our approach are as follows: (1) We have produced the first emulator for a complex plasma transport code. (2) Our method exactly interpolates the results of available code runs for a deterministic code. It also properly accounts for any random (e.g. Monte Carlo) error in a code's output. (3) Using a method called "data adaptive sequential optimization," we have achieved an estimated additional several-fold decrease in the nurnber of transport code runs needed to achieve a given accuracy.

The basic method used to accomplish these results consists of several steps. (For more details, see $[2,3]$.) First, the input-output relation defined by the code in question is assumed to be the sum of two parts. The first part is a linear regression of the output upon the inputs. The second part is a random process. Conceptually, the deviations of the set of all compute code outputs from the best linear regression are considered to have a given statistical distribution, of which our particular code (BALDUR) is taken to be a random sample. (A typical alternative to our method is to assume the deviations from a linear regression of the output upon the inputs are due to quadratic and possibly higher order terms, and then use a technique such as projection pursuit regression to determine which of these many additional terms needs to be included. This alternative only exactly interpolates the code results if the same number of 
terms as data points is included. Moreover, the inclusion of nonlinear polynomial terms tends to lead to large fitting errors near the boundaries. This be undesirable for work where the basic purpose is to calibrate models which have a physical basis that allows them to be extrapolated beyond the boundaries of the region presently being analyzed.)

A simple example, typical of the fitting accuracy of our procedure for the higher dimensional input parameter spaces of interest here, is shown in Fig. 1. For this example, the "code emulator" is given only the output of a function evaluator at ten points. The fit it prociuces, given by the smooth curve, reproduces the actual input-output relation of the function evaluator (cusped curve) with a maximum error of about $10 \%$ and a root mean square error of about $3 \%$. Having deduced a comparable error for our BALDUR simulator (using the method described below), we are satisfied that the relatively small loss in accuracy produced by this procedure is well worth it for the orders of magnitude decrease in computation time needed for our later applications.

The second step in constructing our code emulator is to pick a random sample of inputs. These inputs are chosen from a region defined by the machine operation ranges covered by the experiment, and by a priori estimates of the allowable range of adjustable modelling parameters defined by consultation with the authors of various theories. The regression and statistical parameters in the best linear unbiased predictor ("BLUP") of the code outputs are then varied to maximize the likelihood that the deviations from the resulting linear regression resulted from sampling a random distribution. This well defined process, whose mathematical formulation and proof are documented elsewhere [3], gives an initial code emulator.

The third step is to use the initial code emulator to define an input space sampling procedure which picks five new sets of input parameters. Using a Monte Carlo sampling of all such allowed new parameter sets, it is possible (in the limit of a large sample) to find best sets of new inputs. These inputs are chosen to optimize accuracy of the code emulator expected to be produced when the emulator construction procedure is repeated with the results of the five new code simulations included.

Fourth, the code is run for the five new inputs just selected. A new code emulator is obtained using all the code input-output results 


\section{Example Trpical of Fitting Accuracy}

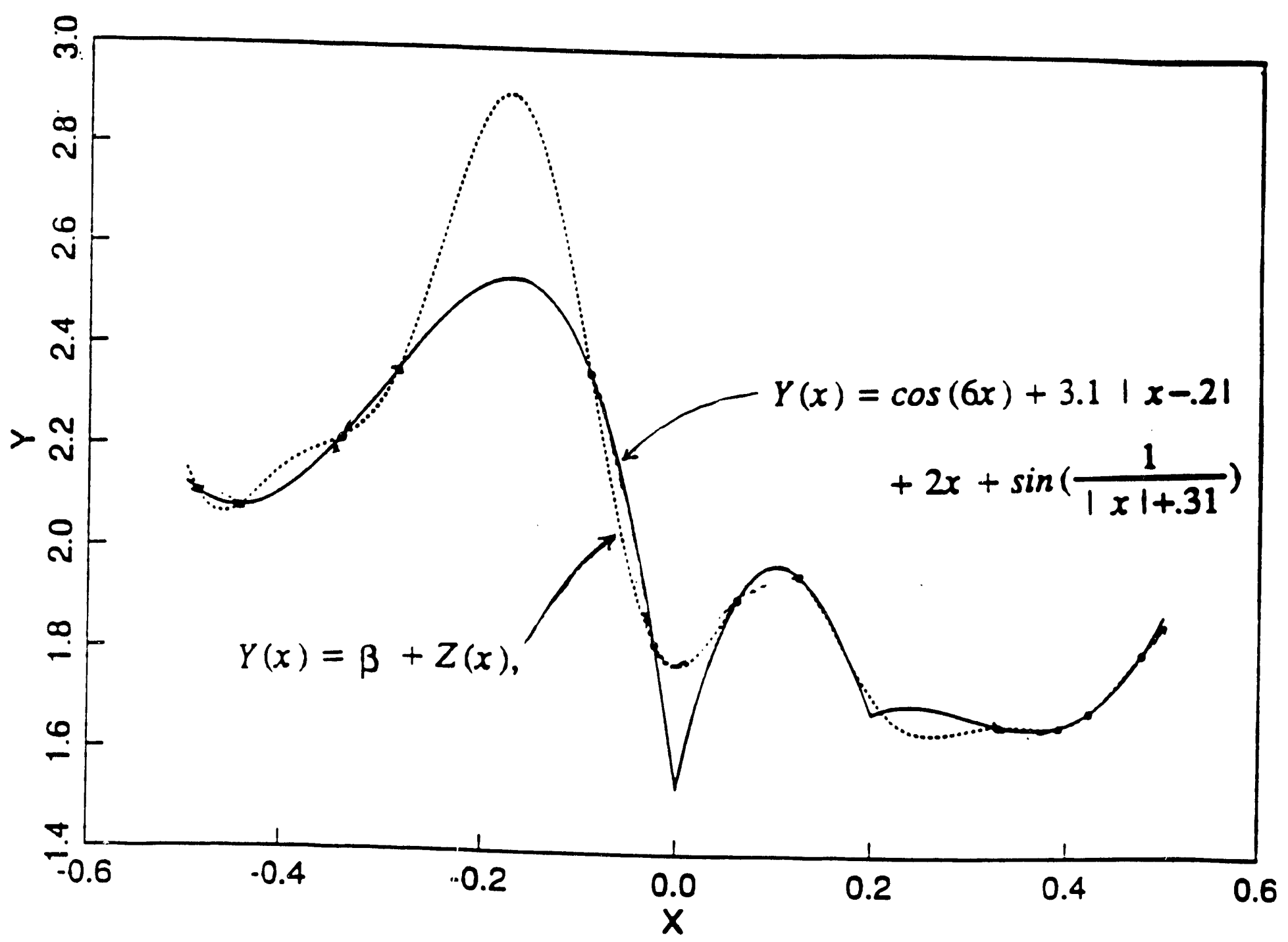

Fig. 1. Example typical of code emulator accuracy: Data at the dots calculated from the indicated function (solid line) was fit with a constant plus a random function (dashed line). 
collected so far. New code emulators are also constructed using all but one of the results obtained so far. The estimation error of each of these code emulators for each omitted point are computed. The root mean square error thereby obtained is then computed. The entire process is then repeated until enough code runs have been accumulated to make this estimated error acceptably small.

As an example application, code emulators for global energy confinement times have been obtained for two tokamaks. The machine parameters to be varied for each tokamak covered the range of parameters found in a data base generously provided by Stan Kaye. These data are meant to be obtained near steady state. We took care, however, to account for small effects of sawtooth oscillations and minimize errors due to possible incorrect simulation of startup. Thus, full time dependent simulations based on well doct!mented discharges with parameters near the centroid of the data base's parameters were used to approach the times at which confinement data are typically obtained. Theoretical modelling parameters varied included three particle/energy flux coefficient multipliers, varied over an order of magnitude range centered near the nominal order-of-magnitude estimates provided by authors of the theories. The threshold for onset of enhanced ion energy fluxes, $\eta_{i t h}$, for $\eta_{i}=d \ln T_{i} / d \ln n_{i}$ was also varied over the range $1 \leq \eta_{i t h} \leq 2$. This covered the range of $a$ priori uncertainty current at the time the emulator construction was initiated.

One minor complication in applying the above procedure to the BALDUR code is that the available configuration of this code produces Monte Carlo noise from the gas transport and neutral beam deposition algorithms. The impact of this on global energy confinement times is small. However, some such impact is unavoidable because, for example, the transport coefficients in detailed theoretical transport models invariably contain terms which depend on the density profiles computed using the Monte Carlo source algorithms. To deal with this problem, an additional zero-mean uncorrelated random error was allowed for in the code emulator construction, and the variance associated with this distribution was estimated simultaneously with the other parameters. As a result, the code emulator for the particular application shown here does not exactly interpolate the code simulation results, except in the limit where the code's Monte Carlo computation error vanishes. Instead, it interpolates the mean which 
would be expected if repeated calculations were made for each input parameter set with a different Monte Carlo seed used in the transport code. This is a highly desirable property. For to exactly interpolate outputs from nearly identical inputs when the output contains a random error would lead to a widely oscillating emulator function. The resulting scatter of code outputs around the code emulator result for two different tokamaks is shown by the circles in Fig. 2. Here, the logarithm of the ratio of code/emulator outputs is plotted as a function of the logarithm of the emulator output for two tokamaks. The meaning of the crosses is defined below. It apparent after this definition that the presence of Monte Carlo variance in the plasma transport code's input-output relation has negligible impact on the overall results.

\subsection{Confidence Contours for Theory Models}

Using the code emulator discussed above, we have computed confidence level contours for the modelling parameters which correspond physically to turbulence saturation amplitudes [3]. This result is shown in Fig. 3. The horizontal and vertical axes on this figure are multipliers of the nominal drift $/ \eta_{i}$ and resistive ballooning driven fluxes originally constructed in consultation with theorists. The ellipses represent a multivariate normal approximation to regions within which there is a $90 \%, 95 \%$, and $99 \%$ probability that these parameters lie. As in any scientific study, these confidence levels are contingent upon the context ir. which they are derived. Here this includes the data base, modelling method, and statistical assumptions used. In this case, the data base consists of all the global confinement data from Kaye's original database for the two tokamaks (PDX and ASDEX) for which code emulators are available. The anomalous transport fluxes used were a linear combination of $\mathrm{drift} / \eta_{i}$, resistive ballooning, and rippling modes, with the $\eta_{i}$ threshold adjustable in the range $1 \leq \eta_{i t h} \leq 2$. The results in Fig. 3 are given for the maximum likelihood values of the rippling multiplier and $\eta_{i t h}$, both of which had relatively little impact on predicted confinement times.

The rippling mode was included largely because Sheffield had suggested that a few times its nominal flux might be significant under some circumstances [4]. It was subsequently noted by Sheffield and others that an alternative formulation of the rippling flux formulas 


\section{RESIDUALS}

告

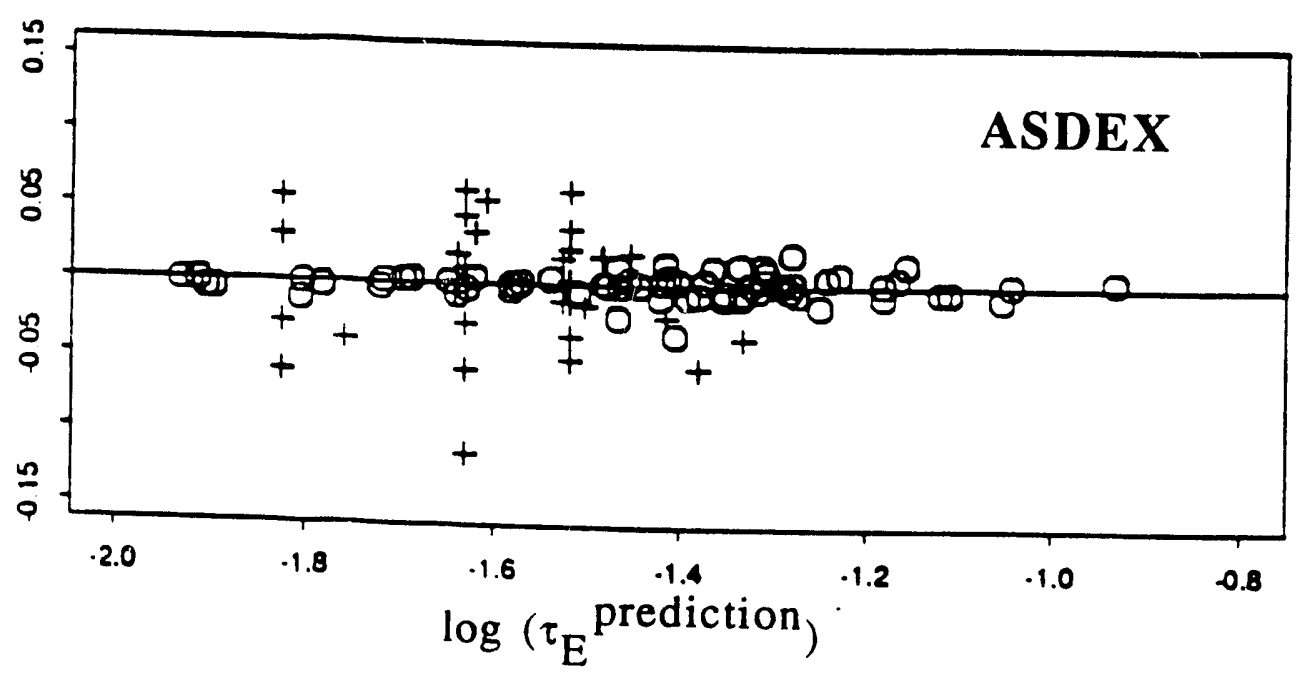

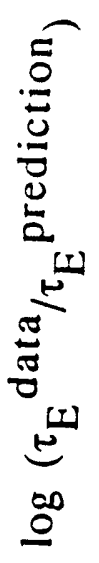

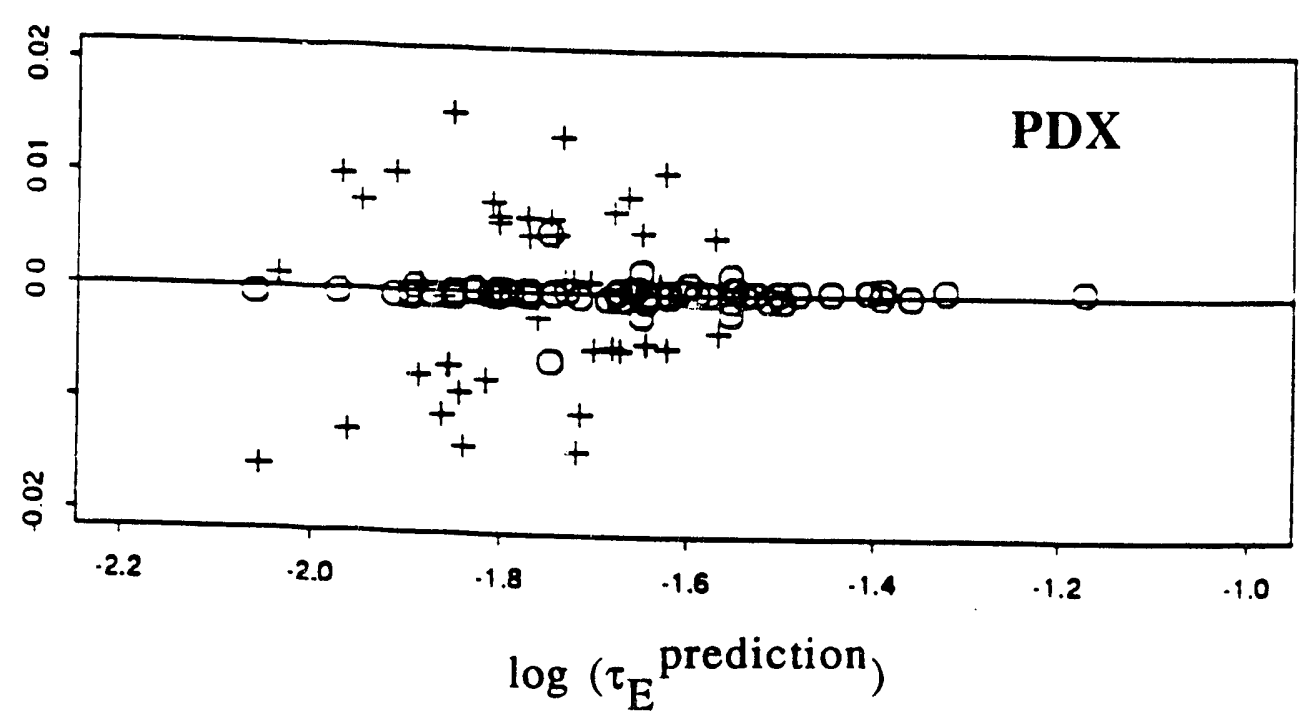

Fig. 2. Logarithm of ratio of code outputs (circles) and experimental data (crosses) to code emulator predictions, plotted vs. logarithm of code emulator predictions. 
Confidence region of $\mathrm{C} 1$ and $\mathrm{C} 3$

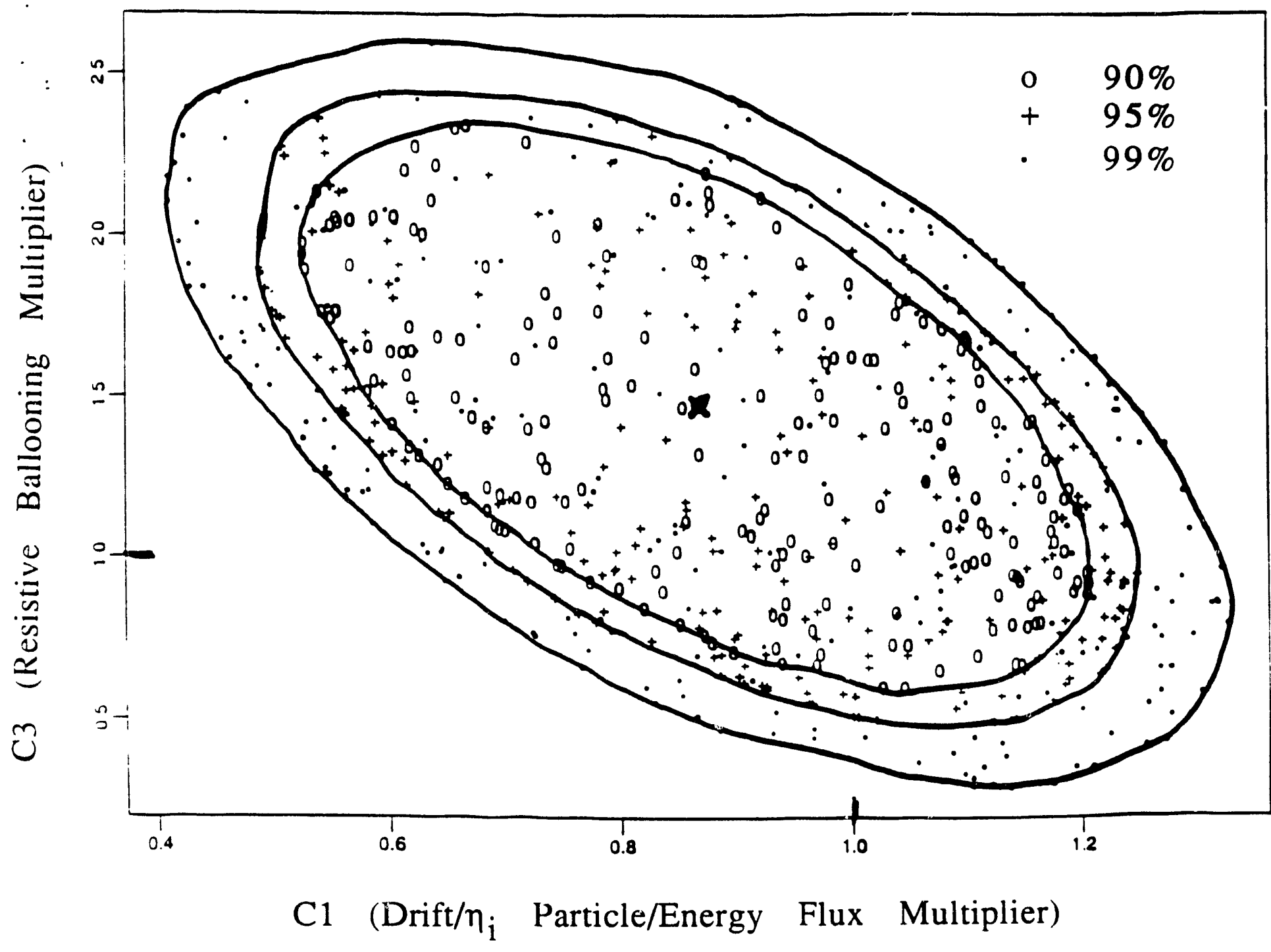

Fig. 3. Confidence contours for $c_{1}$ (mulitiplier of nomindal drift $/ \eta_{i}$ fluxes) and $c_{3}$ (mulitiplier of nominal resistive ballooning fluxes). Symbols indicate points chosen for a Monte-Carlo integration of the probability distribution to obtain the indicated confidence contours. 
would be necessary for this to be the case, at least for the type of discharges investigated here. From the point of view of testing statistical methodology, it is encouraging that the results were consistent with this mode being ne-jigible. To state this more precisely, we showed that no u'seful confidence level could be found for rejecting the "null hypothesis" that rippling mode fluxes are irrelevant to global energy confinement in this large set of L-mode discharges.

In contrast, the results in Fig. 3 indicate that a negligible effect of drift $/ r_{i i}$ or resistive ballooning fluxes can be rejected at a $99 \%$ confidence level in the context of the assumptions used in this exercise. This is consistent with detailed work of Ghanem [5] and others, which showed that using $\mathrm{drift} / \eta_{i}$ fluxes as the only anomaly fails to account for many features of confinement scaling and plasma profiles.

One particularly gratifying aspect of this study is the lack of apparent systematic trends in "residual plots." The crosses on Fig. 2 graph the presumed random distribution of residual discrepancies between the transport model's predictions and the experimental data. Experience in the past using empirical models with many more adjustable coefficients has routinely shown systematic slopes and;or curvature on these plots for various individual tokamaks. We have here an indication that these problems with empirically derived residuals may have been due to inadequacies in the empirical fitting mod. els used, rather than being an indication of fundamental sources of variances which require a much more complicated statistical treatment (cf. [6]).

To summarize work on use of code emulators, we now have every reason to believe that the approach adopted here can lead to systematic construction of the confidence regions needed for testing various transport models against confinement data. A high priority is to extend the basic method illustrated here to a much broader data base (including profile and $\mathrm{H}$-mode data) and a wider class of transport models (c.f. Section 2.6, ielow). The work described next also indicates that we may need to do numerical integration of confidence contours (e.g. using Monte Carlo methods to sum over a set of points such as those indicated in Fig. 1). 


\subsection{Improved Statistical Methods}

For simplicity in an initial study, the work just described was restricted to two rather similar tokamaks, and all of the data was pooled uniformly to obtain the final confidence contours. However, there have long been indications that uniform pooling of all data might be an adequate statistical methodolngy. In our original application for this grant, it was noted that experimentalists and modelers may tend to work on reducing systematic errors in diagnosing and modelling various tokamaks down to roughly the level of random variations in measurments from a given device. The presumed reason for this is that a great deal more vork is often required for what appears to be a rapidly diminishing return if one tries to further reduce systematic errors. Therefore, we constructed a statistical model which allowed for "calibration" discrepancies between transport model predictions and true physical values. In our present analysis, calibration adjustment factors are fixed for a given tokamak but may vary randomly with variance $\sigma_{c}$ from one tokamak to another [i]. For the data from any given tokamak, there are also random "measurement" discrepancies, with variance $\sigma_{m}$ between the best model predictions and reported data. A standard log-linear regression formula of the type used earlier by Kaye and Goldston was consistent with an a posterior mean estimated ratio $\rho=\sigma_{c} / \sigma_{m}$ of $\rho=0.9$ (i.e. $\sigma_{\mathfrak{c}}$ and $\sigma_{m}$ comparable, as postulated). Thus, unless we finc particular reasons not to, we intend to keep at least this level of sophistication in our future analyses of multitokamak data bases.

The same study just referred to also showed that a multivariate normal approximation to the final probability distribution did a very poor joh of reproducing numerically integrated confidence contours. Thus, we also expect to eschew dependence on the common Gaussian approximation to deduced distribution functions. Irstead, we expect to use other functional fits and/or direct Monte Carlo integrations for computing confidence levels and testing theoretical transport models.

\subsection{Profile and Confinement Scaling Studies}

As noted above, methods for construction and use of code emulators and improved statistical methods have been derived and tested against large data bases. We are, therefore, now in a position to test transport theories against very large data bases. To prepare for the 
next round of such studies, we have been exploring the properties of theory refinements which have become available since our original code emulators were constructed. The refinement most directly relevant to our original set of models is an update of resistive ballooning transport flux formulas, as received from B. Carreras (cf. [8]. In particular, the inclusion of $\mathbf{E} \times \mathbf{B}$ effects in this theory has turned out to fill an important gap in particle flux formulas. This gap was not obvious in our previous studies of plasma near transport equilibrium. However, it becomes particularly obvious for $\mathrm{H}$-modes, and for other density-ramped discharges which are fairly opaque to incoming neutrals.

An extensive study of simulations with this model refinement. was further extended, concluded, and documented during the past year [5]. This study included detailed studies of ohmic discharges in ALCATOR-C and ASDEX, as well as extensions into the auxiliary heated ph se in TFTR. DIII-D, ASDEX, PDX, and ISX-B. In a separate project, described below, discharges from DIII, DIII-D, ASDEX, and JET L- and H-modes were simulated. Here there is only room to summarize the basic results of this study.

\subsubsection{Inadequacy of Drift $/ \eta_{i}$ Fluxes Alone}

It was shown definitively that quasilinear $\mathrm{drift} / \eta_{i}$-driven fluxes as the only anomalous transport mechanism are inadequate in the context of our model to reproduce temperature profiles, density profiles, and energy confinement scalings. This is a well known result, but it has rarely been adequately documented in the context of predictive simulations.

\subsection{2 $\tau_{E}\left(\bar{n}_{e}\right)$ in Ohmic Discharges}

It was shown that about half of the saturation of global energy confinement time with increased line-average density in ohmically heated discharges is due to resistive ballooning fluxes in the context of our model. Previously, this effect had been widely thought to be due primarily to $\eta_{i}$ modes. 


\subsubsection{Deviations from Log-linear L-mode $\tau_{E}$ Scalings}

Our previous work showed that the same model which reproduced temperature and density profiles in the above-mentioned tokamaks also reproduced all but perhaps the toroidal field scaling of global energy confinment time deduced from an e.rtire L-mode data base [10]. This work has now been extended to full scans of global energy confinement vs. parameters varied experimentally on each machine. Three important results were found in this stedy.

First, the model reprodu ed the experimentally derived observation that scaling of global energy confinement with plasma current is stronger in smaller machines like ISX-B than in larger machines like TFTR. In the framework studied here, this is because resistive ballooning fluxes tend to be more important in small machines like ISX-B. In larger machines like TFTR, higher temperatures tend to lead to relatively more important $\mathrm{drift} / \eta_{i}$ fluxes. Since the improvement of confinement time with increasing plasma current comes from suppression of resistive ballooning fluxes in this work, it is understandable that there would be a machine-dependent scaling of $\tau_{E}$ vs. plasma current.

Second, this study pointed out the relative paucity of data on machine-dependence of global energy confinement scaling with variations in toroidal field only, at least from analyses of the original L-mode data base. (This mas continue to be a significant question, as recent data from JET indicate a more favorable dependence of $\tau_{E}$ on toroidal field than has been inferred from the entire data base.) This suggests that careful statistical studies may be required to determine whether factors sucii as the safety factor dependence of nominal theoretical transport fluxes need modification for compatibility with available data (c.f. $[8,9]$ ).

Third, our studies showed some obvious deviütions from power law dependence of confinement time on experimental parameters. The most dramatic of these were saturation of confinement time at high density and current in beam-heated L-mode discharges. The densi,y saturation effect is similar to that for ohmic discharges at intermediate densities, augmented by beam-blocking effects. The saturation of confinement improvement at very high plasma current results from a combination of diminishing returns after resistive ballooning fuxes are suppresses and the growth of the sawtoothing region at very high currents. Such a saturation effect has also been 
known for scme time to be possible, from work on DIII.

\subsubsection{Profiles}

Using the present model, we have now thoroughly documented the fairly wide range of L-mode plasma profiles which can be accurately reproduced with only one explicitly adjustable parameter set to a single constant value throughout the study (c.f. detailed results in [5]). There remains, however, an increasingly urgent need from the confinement systems design and boundary plasma studies points of view to obtain boundary conditions models with a sounder theoretical basis for doing extrapolations and understanding the boundary plasma in detail.

\subsection{Boundary Conditions and $\mathrm{H}$-modes}

We had the opportunity during the course of this work to check the standard theoretically motived empirical boundary conditions model used throughout [11]. This model was found adequate for al! cases, except for the boundary density in a ramp to very high density in ISX-B. Systematic use of this model also gave a much better fit to the dependence of $\tau_{F}$ on line average density for low density ohmic discharges. For more detailed future simulations of experimental data, we are thus confident that it will be adequate to calibrate the edge densitites and temperatures at the time point(s) where profiles are reported, using the boundary conditions model only to provide timedependent boundary conditions. Moreover, very modest adjustments of our standard boundary conditions model are likely to be required. A preliminary survey of the requirements for improving our boundary conditions model has been carried out. This survey will be discussed in an accompanying project continuation proposal.

In a separate project, we have also made a study of the evolution of $\mathrm{H}$-mode profiles using a similar transport model [13]. The primary result of this study is that a modest ( $\leq 4$ fold) enhancement of nominal $\mathbf{E} \times \mathbf{B}$ resitive-ballooning-driven particle fluxes (and no "anomalous pinch") is needed to reproduce $\mathrm{H}$-mode density profiles in the present context. It was also found that switching to the collisionless drift formulation at a slightly lower collisionality produced a better fit to JET temperature profile data with little impact on the simulations of other tokamaks performed so far. 


\subsection{Theoretical Flux Formulas}

To prepare for a new round of theory model calibration and testing, we have also updated a previous compilation [14] of encodable fluxsurface-averaged energy and particle flux formulas. A useful feature of this compilation is that results from a ariety of authors are combined in a unified and self-consistent way. Nominal reference values for the next round of model testing are given for parameters not exactly predetermined by existing theory. Instructions are included for including versions of transport fluxes which differ from this reference point. More importantly, suggested prior probability distributions for a priori uncertain theory parameters are given in order to help users of this formulary narrow the regions of parameter space that need to be searched [8].

\section{Conclusion}

It should be clear from the attached summary that a great deal of progress has been made in the past year for a project of this scope. This work is documented in $\mathrm{PhD}$ theses and publications describing statistical and modelling work $[3,5,2,12]$. Major extensions of another article have been submitted for publication [ $[$ ], and a laboratory report on revised theoretical particie fluxes has been circulated for comment [8]. In addition, a considerable amount of exploratory work has been done in preparation for future studies.

Various above-mentioned improvements in analysis methodology and our understanding of the implications of transport theories are particularly important for future work. These are as follows: (1) Theorybased transport models with a very small number of adjustable parameters can reproduce a wide variety of profile and confinement data from ohmic, L-mode, and H-mode discharges. (2) Apparent variations in confinement scaling exponents from one tokamak to another may be largely due to understandable properties of underlying transport mechanisms, rather than due to random variations which can only be treated by an empirical statistical procedure. (3) Remaining discrepencies from results on various tokamaks can be usefully treated using the physically meaningful hypothesis that "calibration" errors in experimental data and modelling procedures vary randomly from one tokamak to another. (4) While our boundary conditions model 
needs improvement to obtain fully predictive tokamak simulations, it is adequate for studies based on existing data bases. (5) Use of optimized predictive transport code emulators allows systematic survey a modest number of a priori unknown parameters in theoretical transport models. This is possible even when the data base used to calibrate and test the theories is very large but contains data (like global energy confinement times) which can not support standard "data analysis" procedures.

Thus, we are now in a position to undertake systematic studies of an expanded set of recent transport theories. These studies should include systematic calibration, confidence contour calculation, and model testing. These studies should be based on a combined profile and confinement time data base of much broader scope than it has heretofore been practicable to use in such a systematic manner. 


\section{References}

[1] B. J. Braams and K. Lackner, "A Proposed Method for Fast Determination of Plasma Parameters," Report IPP 1/228, MaxPlanck-Institut für Plasmaphysik (1984).

[2] D. Cox, J. S. Park, J. Sacks, and C. Singer, "Tuning Complex Computer Code to Data," Proc. Interactions Meeting (Seattle, 1991) in press.

[3 J J. S. Park, "Tuning Complex Computer Codes to Data and Optimal Designs," University of Illinois at Urbana-Champaign Department of Statistics PhD Thesis (1991).

[4] J. Sheffield, Nuclear Fusion 29 (1989) 1347.

[5] E-S. Ghanem, "Theoretical Transport Model for Tokamaks," University of Illinois at Urbana-Champaign Department of Nuclear Engineering PhD Thesis (1991).

[6] P. J. McCarthy, K. S. Riedel, O. J. W. F. Kardaun, H. Murmanu, K. Lackner, the ASDEX Team, "Scalings and Plasma Profile Parameterisation of ASDEX High Density Ohmic Discharge:, Max-Planck-Institut für Plasmaphysik Report IPP 5/34 (1990).

[7] T. Djemil, C. Singer, and D. Cox, "A Bayesian Analysis of Multisite Tokamak Confinement Data," revised for Technometrics (28 June, 1991).

[8] C. E. Singer and G. Bateman, "A Review of Multimode Predictive Tokamak Simulations," University of Illinois Fusion Studies Report FSL-333 (1991).

[9] A. Beklemishev, "Anomalous Ion Thermal Diffusion from $\eta_{i}$ Modes," Bull. Am. Phys. Soc. 36 (1991) 2454.

[10] C. E. Singer, E-S. Ghanem, C. E. Singer, G. Bateman, and D. P. Stotler, Nuclear Fusion 30 (1990) 1595.

[11] C. E. Singer, G. Bateman, and D. Stotler, "Boundary Conditions for OH, L, and H-mode Simulations," Princeton Plasma Physics Laboratory Report PPPL-2527 (1988).

[12] C. E. Singer, W. Choe, D. Cox, et al., "Predictive Modelling of Tokamak Transport," Proc. Thirteenth Int. Conf. Plasma Phys. Controlled Fusion (Washington, 1990), paper IAEA-CN-/D-4-3. 
[13] J. E. Kinsey, "H-mode Transport of Tokamak Plasmas," Iniversity of Illinois Department of Nuclear Engineering Master's Thesis (1991).

[14] C. E. Singer. "Theoretical Particle and Energy Flux Formulas for Tokamaks." Comments Plasma Phys. Controlled Fusion 11 (1988) 165. 

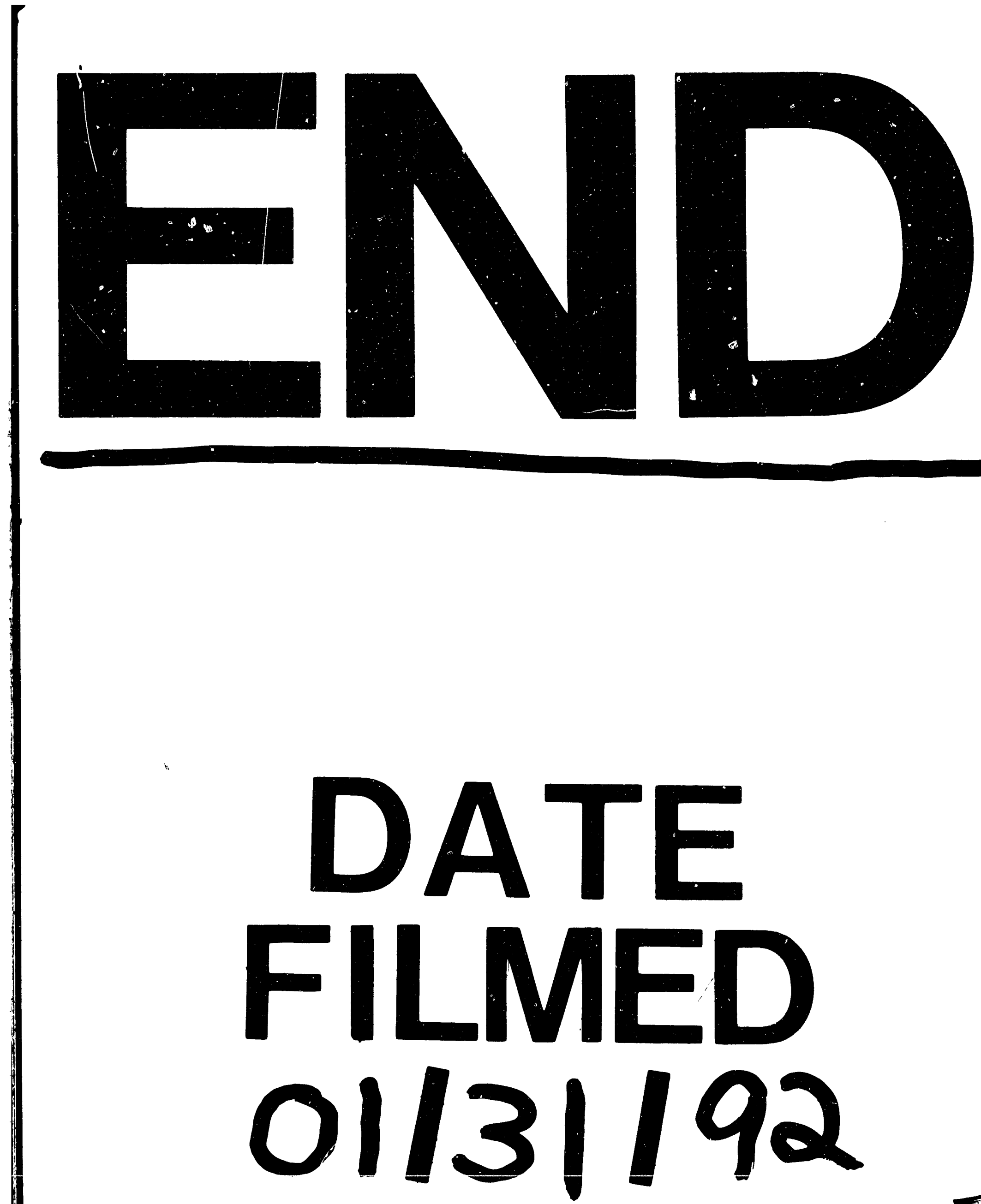
